\title{
Multiscale analysis of friction behavior at fretting interfaces
}

\author{
Zhinan ZHANG ${ }^{1, *}$, Shuaihang PAN ${ }^{2}$, Nian YIN ${ }^{1}$, Bin SHEN ${ }^{1}$, Jie SONG ${ }^{3}$ \\ ${ }^{I}$ State Key Laboratory of Mechanical Systems and Vibrations, Shanghai Jiao Tong University, Shanghai 200240, China \\ ${ }^{2}$ School of Mechanical \& Aerospace Engineering, University of California Los Angeles, Los Angeles 90095, USA \\ ${ }^{3}$ Institute of Nano Biomedicine and Engineering, Shanghai Jiao Tong University, Shanghai 200240, China \\ Received: 20 June 2019 / Revised: 19 September 2019 / Accepted: 13 November 2019 \\ (C) The author(s) 2019.
}

\begin{abstract}
Friction behavior at fretting interfaces is of fundamental interest in tribology and is important in material applications. However, friction has contact intervals, which can accurately determine the friction characteristics of a material; however, this has not been thoroughly investigated. Moreover, the fretting process with regard to different interfacial configurations have also not been systematically evaluated. To bridge these research gaps, molecular dynamics (MD) simulations on $\mathrm{Al}-\mathrm{Al}$, diamond-diamond, and diamond-silicon fretting interfaces were performed while considering bidirectional forces. This paper also proposes new energy theories, bonding principles, nanoscale friction laws, and wear rate analyses. With these models, semi-quantitative analyses of coefficient of friction $(\mathrm{CoF})$ were made and simulation outcomes were examined. The results show that the differences in the hardness, stiffness modulus, and the material configuration have a considerable influence on the fretting process. This can potentially lead to the force generated during friction contact intervals along with changes in the CoF. The effect of surface separation can be of great significance in predicting the fretting process, selecting the material, and for optimization.
\end{abstract}

Keywords: molecular dynamics simulation; friction; wear; fretting

\section{Introduction}

Crystalline materials, such as metallic crystals and atomic crystals, can have a broad niche of applications for their distinctive properties. For example, aluminum (Al) is a typical and important metallic crystal that is often used as the base material in many essential parts in spacecraft [1-4], automobiles, and electronics such as batteries and triboelectric nanogenerators $[5,6]$. This can be attributed to the high performance, good utility, and relatively low cost of aluminum. On the other hand, diamond and silicon (Si) are two traditional materials with high hardness atomic crystals. They are also commonly used in metal processing, coating, and protection $[7,8]$. These materials, owing to their small-scale surface roughness $(1-1,000 \mathrm{~nm})$, inevitably suffer from micro-scale motions, i.e., the fretting process, when they form interfaces under normal service $[9,10]$.

Theories such as the stick-slip effect $[11,12]$ can help in the understanding of the fretting phenomena. However, the fretting process for $\mathrm{Al}, \mathrm{Si}$, and diamond, until recently, has not been fully understood by the characteristic interface configuration. For example, Li et al. [13] and Lan et al. [14] analyzed the friction phenomena of Si incorporated diamond-like carbon films. Although the idea is useful for complex systems, it ignores how dissimilar materials and their signature properties are related during the friction process. Thus, it is not suitable to explain the universal fretting processes.

Moreover, friction contact intervals exist as the counter-part of the friction contacts. This is because the friction heat dissipation needs a relaxation period

* Corresponding author: Zhinan ZHANG, E-mail: zhinanz@sjtu.edu.cn 
[15-17]. Friction contact interval refers to the time period when a certain asperity does not directly contact with or becomes severely deformed by the countersurface asperities and when the force response shows less surface-interactive features. However, in most studies, the key feature of friction contact intervals is ignored. For instance, although the work by Morita et al. [18] and Sha et al. [19] emphasized more on the fundamental effects of a single material or chemical bases and groups; they merely focused on frictional contacts.

In comparison to traditional research methods, e.g., finite element analysis, molecular dynamics (MD) simulations have an extremely high spatial and temporal resolution. MD simulations reveal the atomic scale friction by considering the macroscopic Hertz theory with an adhesion contact (e.g., JKR model (discovered by JOHNSON K L, KENDALL K, and ROBERTS A D) and DMT model (discovered by DERJAGUIN, MULLER, and TOPOROV)) [20]. To gain more insights into fretting friction, MD simulations were successfully applied to investigate the mechanism of monoatomic layer removal, abrasive rolling effects, material removal, and the surface finish in chemical or mechanical polishing processes [20-22]. However, questions remain in terms of how soft and hard materials (e.g., metals and atomic crystals) behave and how the force evolves during friction at an atomic scale are open to debate [23]. As a result, MD may be able to answer these questions. Although the standard tip-shape sliding model for friction analyses is commonly used in MD simulations [24], its relationship with the macroscopic phenomena, such as the coefficient of friction (CoF), is not fully and quantitatively understood [20]. Moreover, a reasonable layer division, a thermal boundary setup, and an asperity geometry, also add to the diversity and complexity of MD solutions for fretting friction problems [20, 25, 26]. As a result, dynamical analyse that can distinguish friction contacts and friction contact intervals are often neglected. Whether the friction contact interval will play a role in MD simulations is unclear.

In this study, MD simulations are performed to examine the whole continuous friction behavior of $\mathrm{Al}$, diamond, and Si fretting interfaces by focusing on a comparison of their force responses. First, by referring to the published simulation parameters [23, 24, 27], the effects of interfacial configurations and material properties were examined by simulating the $\mathrm{Al}-\mathrm{Al}$, diamond-diamond, and diamond-Si fretting interfaces. Second, new theories are proposed to explain the cause of these effects. A good match between the simulation results, the theoretical analysis, and the available data illustrates the feasibility of the MD simulations to link the micro- and macro-fretting friction behavior. The different contributing factors for fretting surface separation, such as simulation conditions, are also considered. In brief, this paper illustrates the multiscale analysis approach by using the sequence of MD simulations to multiscale physical mapping. This includes the Hertz theory and statistical thermophysical laws, which leads to parameter correlations (e.g., $\mathrm{CoF}$ and interfacial separation) in the related fretting friction problems. This approach can guide the rational design of fretting friction systems for broader applications.

\section{Methodology}

\subsection{Fretting layer configuration}

The simulation box is set up in large-scale atomic/ molecular massively parallel simulator (LAMMPS) for a volume of $70 \AA \times 40 \AA \times 52 \AA$ with the use of periodic boundary conditions along the $x$ - and $y$-directions, i.e., pps [28]. There were 7,402 aluminum atoms on the $\mathrm{Al}-\mathrm{Al}$ fretting interface, 21,717 carbon atoms for the diamond-diamond fretting interface, and 3,112 silicon atoms with 10,844 carbon atoms for the diamond-Si fretting interface. The relative velocity of the two fretting interfaces was set to $0.1 \AA / p$ s for continuous frictional contact. An illustration is shown in Figs. 1(a) and 1(b).

Here, the two fretting interfaces are separated by a constant distance of $12 \AA$ to mimic the real working conditions. This is achieved by supplying the equivalent vibrational loads during minute fretting motion while simplifying the simulation by simulating the fretting stroke via the periodical frictional contact process [29]. The selected separation guarantees that there will be no force interaction for the upper and lower substrates. The surface roughness is a factor that highly affects the performance of the two fretting interfaces [30,31]. Therefore, when the analysis is 


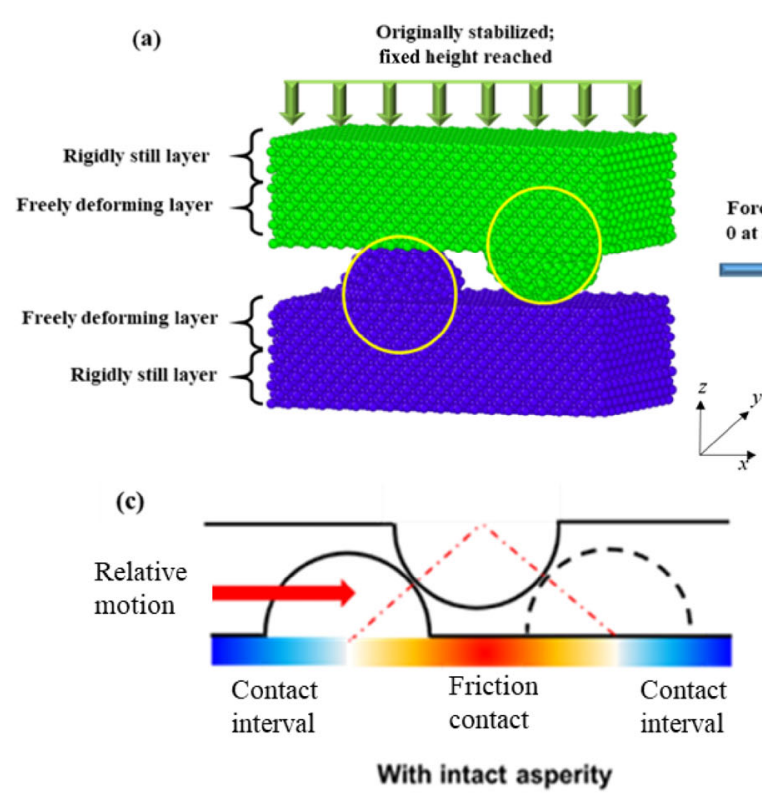

(b)
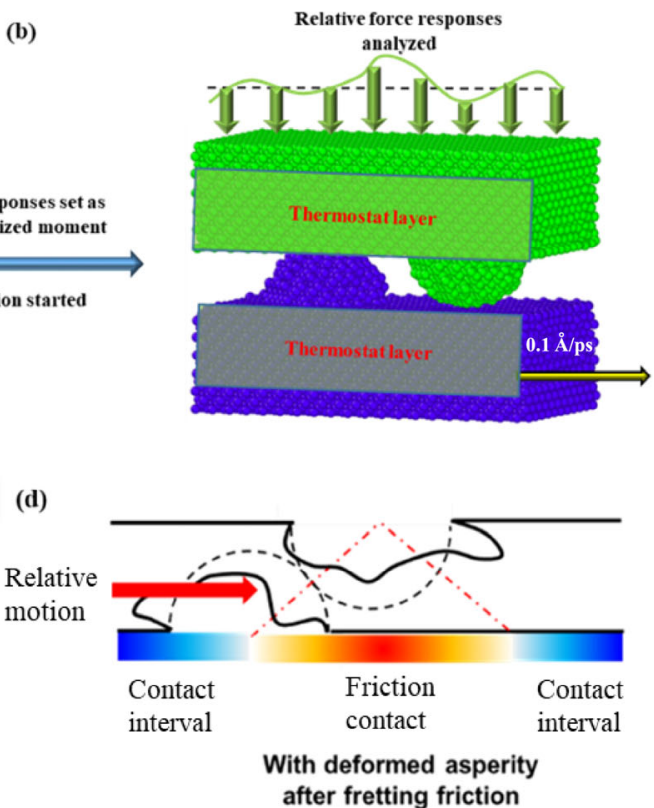

Fig. 1 Illustration of the MD simulation of the fretting interface and friction process: (a) the fretting interfaces model and simulation settings, (b) simulation conditions when the motion is started (diamond-diamond fretting interface as an example)/illustration of the friction contact intervals, (c) with intact asperities, and (d) contact surface deformation after the fretting process.

focused on micro principles, it is reasonable to simulate the single asperity in a hemisphere shape [32]. Because a single asperity size will affect the friction behavior between the fretting interfaces, the single asperity was set to $24 \AA$ in diameter for the closest contact conditions [24], and two asperities were initially $30 \AA$ apart and free from the molecular force interaction for relaxation, which ensures equilibrium.

Both the upper and lower substrates were divided into two parts according to their motion characteristics. The lower substrate consists of the moving layer (0-6 ̊) and the freely deforming layer (6-20 A). It can guarantee full interaction force relaxation from the influenced or contacted layers. For the upper substrate, the configuration consists of the freely deforming layer (32-46 $\AA$ ) and the immobile layer (46-52 $\AA$ ). A summary of the configuration parameters is listed in Table 1. Both parts of the immobile layers are regarded as rigid bodies. The internal degrees of freedom are fixed with a strong harmonic potential to maintain the shape of the whole system [18]. Moreover, the temperature of the asperities on the upper and lower substrates varies without restriction because the fretting process may introduce heat transfer. The freely deforming layers were simulated at a constant temperature of $300 \mathrm{~K}$ [33].
Table 1 Geometric information of the MD simulation model.

(a) Simulation box illustration

\begin{tabular}{ccc}
\hline Part & Shape & Dimension $(\AA)$ \\
\hline Lower substrate & Cubic & $70 \times 40 \times 20$ \\
Upper substrate & Cubic & $70 \times 40 \times 20$ \\
Single asperity & Hemispheric & $D=24$ \\
\hline
\end{tabular}

(b) Material characteristics parameters

\begin{tabular}{cccc}
\hline Material & Orientation & Lattice $(\AA)$ & Bond length $(\AA)$ \\
\hline $\mathrm{Al}$ & $\begin{array}{c}\text { Face-centered } \\
\text { cubic (FCC) }\end{array}$ & 4.03 & $\mathrm{Al}-\mathrm{Al}: 2.80[25]$ \\
Diamond & Diamond (Cubic) & 3.57 & $\mathrm{C}-\mathrm{C}: 1.54$ \\
$\mathrm{Si-crystal}$ & Diamond (Cubic) & 5.43 & $\mathrm{C}-\mathrm{Si}: 1.85$ \\
\hline
\end{tabular}

The friction contact interval denotes the period for a certain asperity that is not in direct contact with or is severely deformed by the single asperity as depicted in Fig. 1(c). To simplify the analysis after several instances of fretting frictional contact, this definition also applies based on the original geometry as demonstrated in Fig. 1(d) [15]. The method and definition are similar to the fractal model proposed by Chen et al. [15]. This is also compatible with other simulation methods, including finite element analysis, when considering the size effect, the configuration 
shapes, and other factors [34-36]. This contact interval model, which simulates two individual continuously contacted asperities in the fretting process, gives the force response analyses a more dynamical basis on surface evolution with time.

\subsection{Force field selection}

To better describe the material interaction and energy, $\mathrm{Al}$ is described with the embedded atom method (EAM) force field [37-39], which denotes the interactions between metallic atoms:

$$
E_{i}=F_{\alpha}\left(\sum_{j \neq i} \rho_{\beta}\left(r_{i j}\right)\right)+\frac{1}{2} \sum_{j \neq i} \varphi_{\alpha \beta}\left(r_{i j}\right)
$$

Where $E_{i}$ denotes the interaction energy; $F_{\alpha}$ is the embedding function; $\rho_{\beta}$ indicates the electron charge density of atom type $\beta$; $r_{i j}$ is the separation distance between the atoms $i$ and $j ; \varphi_{\alpha \beta}$ is a factor for the pair potential interaction depending on the element types of atoms $i$ and $j ; \alpha$ and $\beta$ are label types of the atomic elements $i$ and $j$.

Furthermore, diamond and Si are described with the Tersoff force field [40-42]:

$$
\begin{gathered}
E=\frac{1}{2} \sum_{i} \sum_{j \neq i} V_{i j} \\
V_{i j}=f_{\mathrm{c}}\left(r_{i j}\right)\left[f_{\mathrm{r}}\left(r_{i j}\right)+f_{\mathrm{a}}\left(r_{i j}\right) \times b_{i j}\right]
\end{gathered}
$$

Equations (2) and (3) describe the interactions between the atoms as three-body interactions with considerations for the repulsive and attractive forces, $f_{\mathrm{r}}$ and $f_{\mathrm{a}} \cdot V_{i j}$ is the interaction potential between atoms $i$ and $j$. It is a function of $f_{\mathrm{r}}$ and $f_{\mathrm{a}}$ and is modified by the ratio $b_{i j}$ and the distance factor $f_{\mathrm{c}}$, which determines the effect of the cutoff distance.

\section{Results and discussions}

\subsection{Fretting process analysis}

\subsubsection{General trend}

During the simulation, the friction and normal force values are important factors that need to be considered. After the simulation was started and when the original system reached equilibrium [29], the force responses were regarded as zero to rule out the Derjaguin effect. The friction and the normal force to friction were treated as relative values to describe the changes during the fretting processes as presented in Figs. 1(a) and 1(b) [43]. As a result, the influence of the forces before equilibrium can be excluded.

The whole fretting process is depicted in Fig. 2. The first friction contact process was investigated for severe wear or deformation, which is presented Fig. 3. During the first friction contact, if the upper and lower single asperities were equally hard and stiff, both individual asperities displayed severe wear from $12 \AA$ to $4-6 \AA$ or less as illustrated in Figs. 2(c), 3(a), and $3(b)$. If the upper and lower single asperities have a large hardness and stiffness differences, which is the case for the diamond-Si fretting interface, the relatively soft asperity will be deformed and will be worn to a mostly flat surface. In addition, the harder asperity will remain intact, as depicted in Fig. 3(c). It should be noted that diamond has a Mohs hardness of 10.0 and a bulk modulus $530.0 \mathrm{GPa}$, while Si has a Mohs hardness of 7.0 and a bulk modulus of 95.0 GPa.

\subsubsection{Difference of atom redistribution/transfer modes}

For $\mathrm{Al}-\mathrm{Al}$ and diamond-diamond coupled fretting interfaces, there is no difference in the hardness between (a)

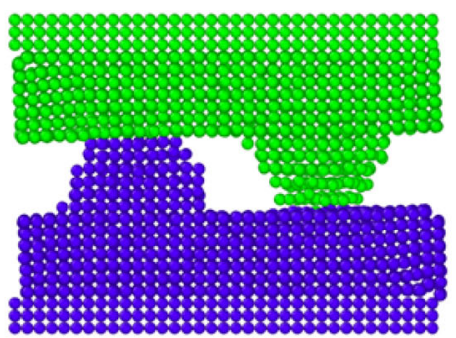

(b)

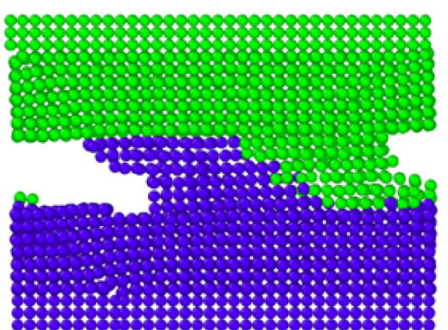

(c)

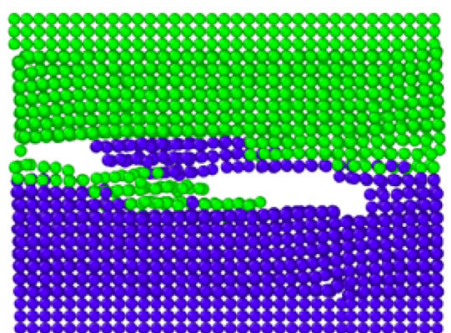

Fig. 2 Illustrations of Al-Al first friction contact during the fretting process: (a) the fretting process starts, (b) wear begins during the first friction contact, and (c) the first friction contact finishes and the interval ends. 
(a)

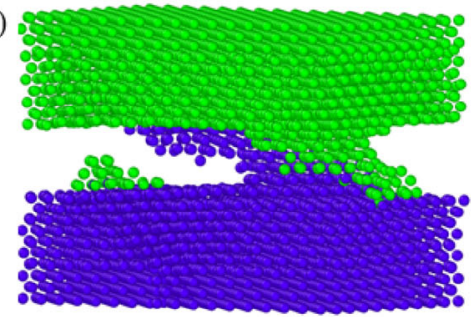

(b)

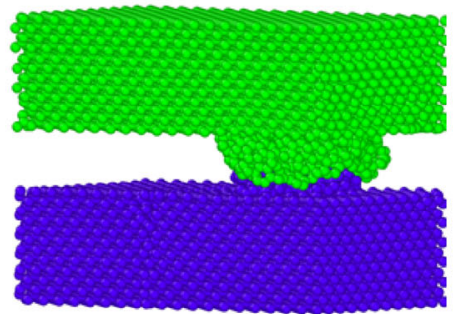

(c)

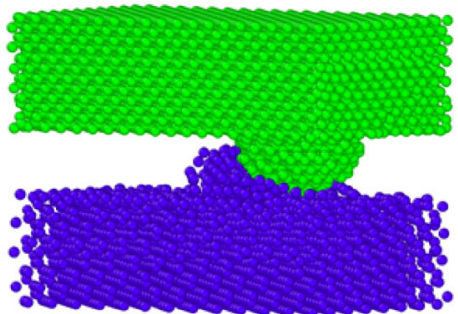

Fig. 3 Single asperity wear situation after the first friction contact for (a) Al-Al fretting interface, (b) diamond-diamond fretting interface, and (c) diamond (upper) $-\mathrm{Si}$ (lower) fretting interface.

the upper and lower substrates. Both asperities show clear signs of wear as indicated by the microscopic mass transfer with collective atomic exchange, which is revealed by the atomic bond analysis. In addition, the contact area increases, especially during the first friction contact as presented in Figs. 2(a) and 2(b). The $\mathrm{Al}-\mathrm{Al}$ interface has a different area increment mode than the diamond-diamond interface as depicted in Figs. 3(a) and 3(b).

Macroscopically, this can be analyzed by the Hertz theory with adhesion conditions [20]:

$$
A \cong \omega \times\left[\frac{R \times\left(F_{N}+4 \gamma \pi R\right)}{E^{*}}\right]^{2 / 3}
$$

Where $A$ indicates the contact area; $\omega$ is the constant factor for the detailed geometric effect; $R$ is the effective asperity radius; $F_{N}$ is the effective normal load; $E^{*}$ is the equivalent contact response factor determined by the Poisson's ratio $v_{i}$ and the Young's modulus $E_{i}$ in terms of $E^{*}=\frac{4}{3} \sum_{i} \frac{1-v_{i}^{2}}{E_{i}}$. In addition, $4 \gamma \pi R$ illustrates the adhesion contact modification, which regards the surface energy $\gamma$ of the fretting surfaces.

Under our simulation conditions, the change in area (increment) will be affected according to

$$
\begin{aligned}
\frac{\mathrm{d} A}{\mathrm{~d} F_{N}} & =\frac{2}{3} \times \omega \times\left(\frac{R \times\left(F_{N}+4 \gamma \pi R\right)}{E^{*}}\right)^{-\frac{1}{3}} \times \frac{R}{E^{*}} \\
& =\frac{2}{3} \times\left.\frac{A\left(F_{N}\right)}{F_{N}+4 \gamma \pi R}\right|_{R, E_{i}, t}
\end{aligned}
$$

Note that the surface energies of $\mathrm{Al}, \mathrm{Si}$, and diamond are of the same order of $\sim 1,000 \mathrm{~mJ} / \mathrm{m}^{2}$. Because the
Al-Al interface has a smaller Young's modulus than the diamond-diamond interface, then the $\mathrm{Al}-\mathrm{Al}$ interface has a smaller force produced with the same initial conditions. Therefore, with a larger area and a smaller force response after the same time period, the Al-Al interface will evolve to obvious wear and a larger increase in contact area according to the implicit function of Eq. (5).

Microscopically, the diamond lattice parameter is less than aluminum as displayed in Table 1. The bond equivalent spring constant, i.e., the stiffness denoted by the bulk moduli, of $\mathrm{C}-\mathrm{C}$ is much larger than $\mathrm{Al}-\mathrm{Al}$. That is

$$
\begin{aligned}
E(\mathrm{Al}-\mathrm{Al}) \cong & \frac{1}{2} k_{\mathrm{Al}} \Delta x_{\mathrm{Al}}{ }^{2} \approx \frac{1}{2} k_{\mathrm{C}} \Delta x_{\mathrm{C}}{ }^{2} \cong E(\mathrm{C}-\mathrm{C}) \\
& k_{\mathrm{Al}}<k_{\mathrm{C}} ; \Delta x_{\mathrm{Al}}>\Delta x_{\mathrm{C}}
\end{aligned}
$$

where $E$ denotes the thermal energy of the atoms at a certain temperature under the harmonic oscillation approximation, and $k$ is the effective spring constant for the atoms. This means that the worn $\mathrm{Al}$ atoms are easier to redistribute in a longer-range and increases the frictional area. In contrast, the worn C atoms depend on the deformation in a shorter-range.

Moreover, $\mathrm{Al}$ as a metallic crystal has homogenous bonding possibilities in all directions because the bonding behavior is dominated by free electrons. Meanwhile, diamond, which is an atomic crystal, is fully bonded with certain orientations by covalent electrons. Given the relationship, the possibility for a diamond atom to transfer will be much lower than an aluminum ion/atom $[20,24,43,44]$, that is

$$
\begin{aligned}
& P(\text { surface }\rightarrow \text { surface }) \propto \\
& \qquad \exp \left(\frac{\sigma \times V-\varepsilon_{\text {bond }} \times N(V)+\Delta \varepsilon \times N(V)}{k_{B} T}\right)
\end{aligned}
$$




$$
P(\mathrm{C} \rightarrow \mathrm{C}) \ll P(\mathrm{Al} \rightarrow \mathrm{Al})
$$

where $P$ (surface $\rightarrow$ surface) denotes the single atom transfer probability due to the bond breaking at the interface. $\sigma \times V$ is the product of the load stress $\sigma$ and the activation volume $V$, and $\varepsilon \times N(\mathrm{~V})$ is the bond deformation energy for the single bond breaking energy $\varepsilon \approx \frac{1}{2} k_{\text {atom-atom }} \Delta x^{2}$, and the number of atoms/ions in the activation volume $N(\mathrm{~V})$ at the detached surface.

As a result, free electrons promote residual stress along the lattice deformation by eliminating the bonding direction trends. This is achieved by spreading the worn atoms more easily and propagating the energy more quickly. On the other hand, diamond atoms can only hold a certain shape, even under severe deformation $[45,46]$.

On the other hand, $\Delta \varepsilon \times N(V)$ denotes the attaching energy $\Delta \varepsilon$ of the same transfer atoms along the attached surface. The attachment of the worn atoms can be divided into two energy forms: direct re-bonding and/or van der Waals interaction. We should note that this volume is different from the single asperity volume. This is because the activation energy also accounts for the volume that is not directly inside the asperity, but is affected by the stress.

Results after three continuous fretting friction contacts are summarized in Figs.4(a)-4(c). The diamond-Si tribo-pair, even after the third fretting contact, is shown in Fig. 4(c); the worn Si and diamond atom cannot attach to the diamond surface. In contrast, the Al-Al tribo-pair displays a clear ion/atom transfer. This is caused by the physical phenomenon described in Eqs. (8) and (9). According to Fig. 4(d), given the tight-bonding, the deformation is due to inter atomic potential barrier reduction, which frees the atoms/ions in the inner-material to move $[47,48]$. However, the attachment of the atom requires the energy barrier to be overcome at the interface, which is proportional to their bond dissociation energy. This is approximately $\sim 186 \mathrm{~kJ} / \mathrm{mol}$ for Al-Al, $\sim 607 \mathrm{~kJ} / \mathrm{mol}$ for $\mathrm{C}-\mathrm{C}$, and $\sim 435$ $\mathrm{kJ} / \mathrm{mol}$ for $\mathrm{C}-\mathrm{Si}$. These energy barriers are due to the different bond lengths as shown in Table 1(b). This energy is much larger than the bond deformation energy as presented in Fig. 4(d).

Because the $\mathrm{Al}-\mathrm{Al}$ bond dissociation energy is smaller than that of $\mathrm{C}-\mathrm{C}$ and $\mathrm{C}-\mathrm{Si}$, it is easier for $\mathrm{Al}$ to cross the interfacial barrier in an atomic heterosurface attachment. This is confirmed by the bonding analyses after each frictional contact, as depicted in
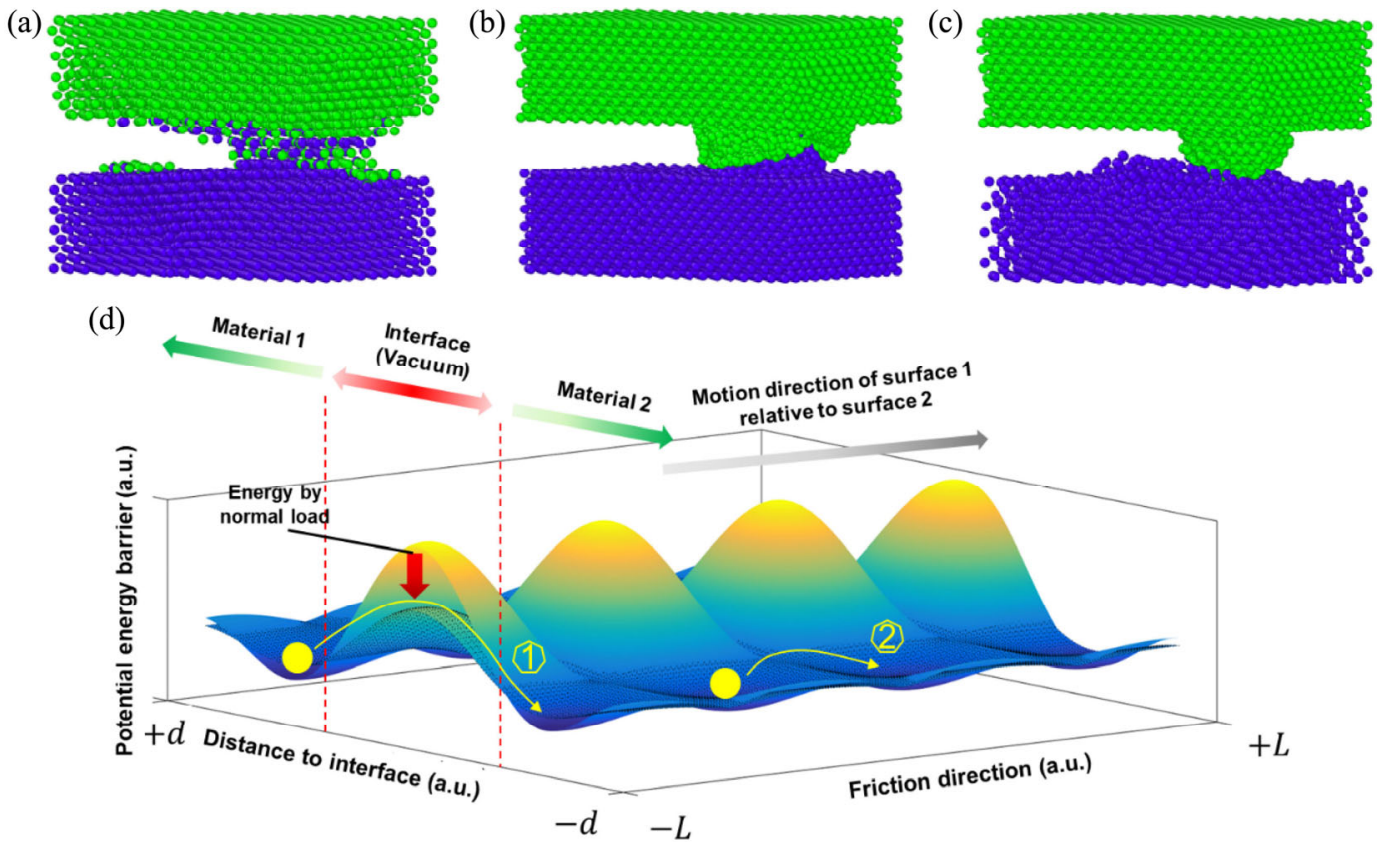

Fig. 4 Single asperity wear situation after the third friction contact for (a) the Al-Al fretting interface, (b) the diamond-diamond fretting interface, and (c) the diamond (upper) -Si (lower) fretting interface, (d) the illustrative description of the atomic-scale deformation (bond deformation energy) and the interfacial attachment (bond dissociation energy)_Process 1: Atom transfer between the surfaces; Process 2: Atom inner-surface redistribution. 
Fig. 5. Even if the van der Waals interaction is considered as the attachment driving force, the attachment probability analyses are feasible. This is because the van der Waals interaction is only $\sim 0.1-10 \mathrm{~kJ} / \mathrm{mol}[49$, 50]. From the simulation, it is reasonable to believe that the atomic hetero-surface attachment is the indicator of an interfacial transfer. This observation is significant for coating, lubricant, and other fundamental tribology studies. This is because these processes and applications involve inevitable interfacial contacts and mass transfer $[18,20]$. Their performance is mainly determined by the evaluation of a similar heterosurface attaching process.

\subsection{Force responses and $\mathrm{CoF}$}

The results of the friction force and the normal force responses are provided in Fig. 6. From the microscopic point of view, the friction force is related to the normal force and the contact area [51, 52]. The estimation for the $\mathrm{CoF}$ is

$$
\begin{aligned}
\bar{\mu} & =\frac{\mathrm{d} F_{f}}{\mathrm{~d} F_{N}} \cong \frac{\mathrm{d} F_{f-\text { force }}+\mathrm{d} F_{f-\text { area }}+\mathrm{d} F_{f}(v, T, \ldots)}{\mathrm{d} F_{N}} \\
& =\mu_{\mathrm{F}}+\mu_{\mathrm{A}}+o(\mu)
\end{aligned}
$$

Equation (10) indicates that friction is composed of two components in the micro-scale. $F_{f \text {-force }}$ or $\mu_{\mathrm{F}}$ demonstrates how the normal force governs the friction force responses; whereas $F_{f \text {-area }}$ or $\mu_{\mathrm{A}}$ clarifies the effect of the contact areas, i.e., the atomic scale frictional adhesion, in the frictional analysis.

One clear observation is that for $\mathrm{Al}-\mathrm{Al}$, there exist obvious and significant force responses in the friction contact intervals. As stated in Section 3.1, it is easier for $\mathrm{Al}$ in hetero-surface attachment. The attachment and the increase in area, as shown in Fig. 4(g), delay the separation of the atoms from both $\mathrm{Al}$ surfaces, which leads to the interval force response. Therefore, we propose that this is the reason why metal surfaces have a higher friction. Besides, the frequent negative force response on the $\mathrm{Al}-\mathrm{Al}$ surfaces indicates the isotropic force exerted by the ions/atoms. This is different from diamond-diamond and diamond-Si surfaces. It also illustrates why past experiments and simulations can only give a wider range of the varying CoF in comparison to two other systems as described in Table 2.

When $\mathrm{Al}-\mathrm{Al}$, diamond-diamond, and diamond-Si fretting interfaces reach an equilibrium, the CoFs can be calculated while considering the fretting intervals as shown in Eq. (10). After referring to the normal CoF range for the above materials, a comparison was made and the error is listed in Table 2 [53]. This provides reasonably well-matched quantitative results. Along with the fretting process analyses and the force response characterizations, this can be helpful for predicting and optimizing the interfacial fretting friction behavior.

\subsection{Effect of separations}

As mentioned above, a single asperity that is $24 \AA$ in diameter and is separated by $12 \AA$ is for the closest contact conditions. However, for this separation, this may not be the worst fretting conditions or the worst friction conditions with the largest $\mathrm{CoF}$. To investigate the effect of the separation distance on the fretting friction behavior, a series of 12,18 , and $24 \AA$ separations were simulated and the results are plotted in Fig. 7.

Because only the $\mathrm{Al}-\mathrm{Al}$ interface encounters severe wear with a clear atomic transfer, the effect of separation

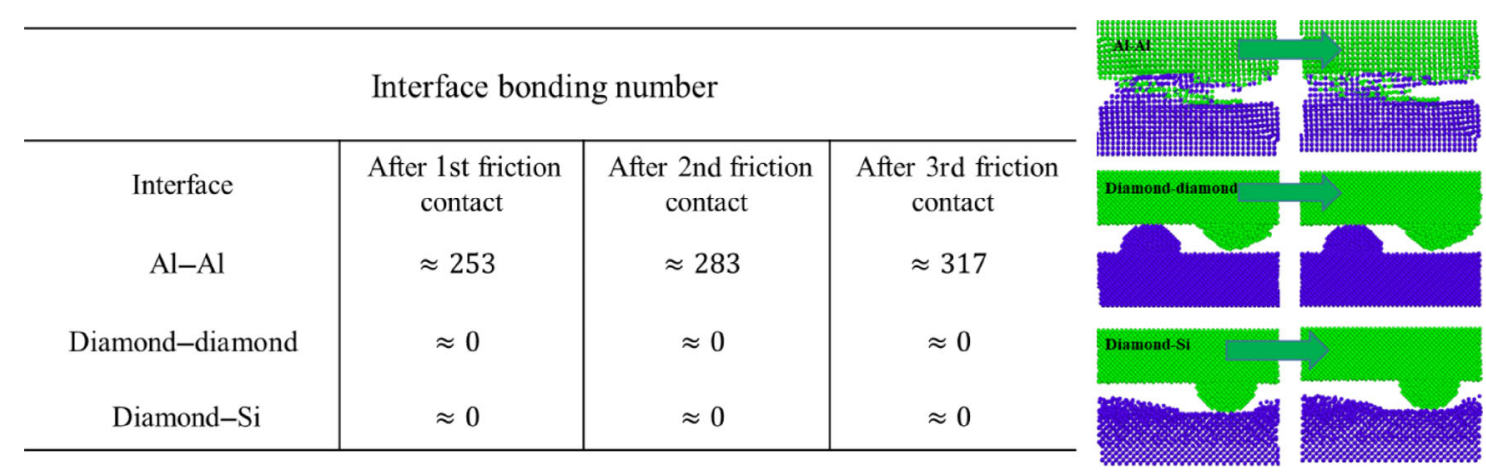

Fig. 5 Results of the bonding analyses for $\mathrm{Al}-\mathrm{Al}$, diamond-diamond, and diamond-Si after each friction contact. 

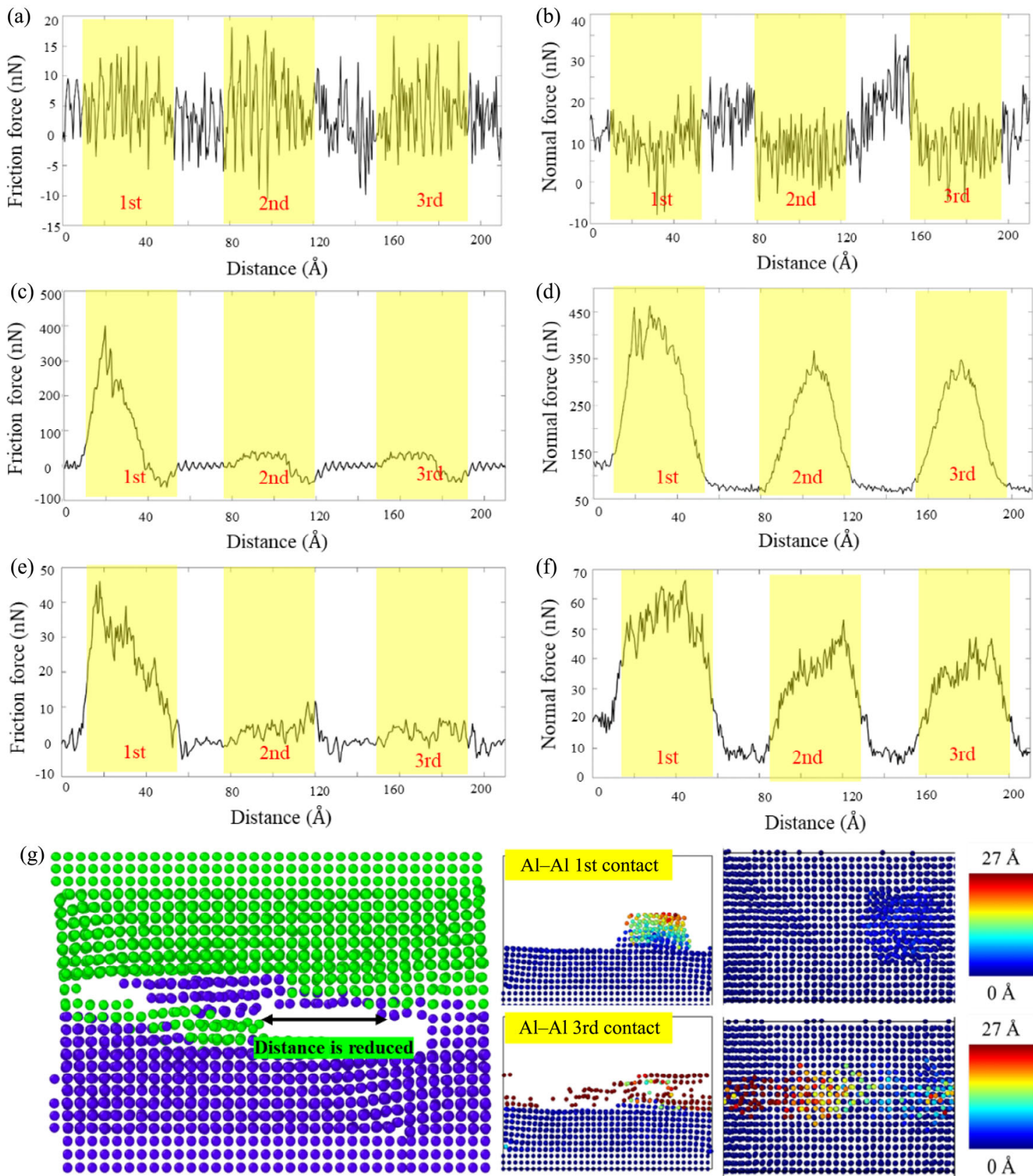

Fig. 6 Force responses between the fretting interfaces during continuous frictional contact: (a) the friction force responses for the Al-Al fretting interfaces, (b) the normal force responses to the friction force for the Al-Al fretting interfaces, (c) the friction force responses for the diamond-diamond fretting interfaces, (d) the normal force responses to the friction force for the diamond-diamond fretting interfaces, (e) the friction force responses for the diamond-Si fretting interfaces, and (f) the normal force responses to the friction force for the diamond-Si fretting interfaces. All non-shadowed areas indicate the friction contact intervals, (g) illustration for the distance reduction and area increment of the $\mathrm{Al}-\mathrm{Al}$ frictional contact, interval force responses, and the displacement of the $\mathrm{Al}-\mathrm{Al}$ lower layer.

Table 2 Comparison of the simulated $\mathrm{CoF}$ and the $\mathrm{CoF}$ range.

\begin{tabular}{ccccc}
\hline Material A & Material B & Friction coefficient range & Simulated CoF & Error \\
\hline Al & Al & $1.05-1.35$ & 1.189 & $\sim 0 \%$ \\
Diamond & Diamond & $0.10-0.16$ & 0.175 & $\sim 9.4 \%$ \\
Diamond & Si-crystal & $0.19-0.33$ & 0.178 & $\sim 6.3 \%$ \\
\hline
\end{tabular}



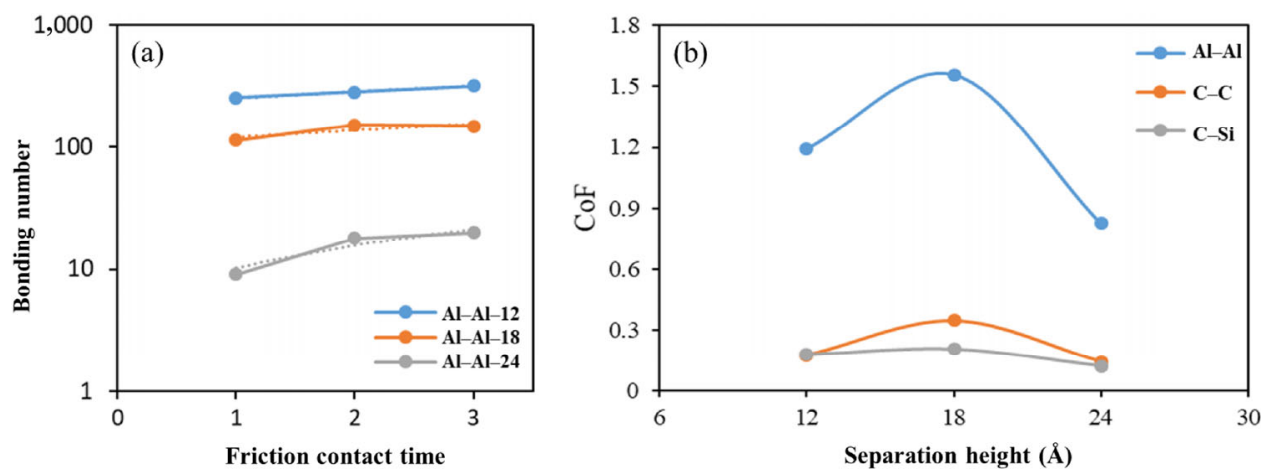

Fig. 7 (a) The worn atomic bonding number of the Al-Al tribo-pairs during the first three friction contacts separated by 12, 18, and $24 \AA$; (b) theCoFs of Al-Al, diamond-diamond, and the diamond-Si tribo-pairs under the separation of 12, 18, and $24 \AA$. Note: The "contact time" here refers to the number of contacts of interests.

on the bonding numbers is investigated for the $\mathrm{Al}-\mathrm{Al}$ tribo-systems. As shown in Fig. 7(a), with the increase in the separation, the bonding number decreases. This is because $R$ in Eqs. (4) and (5) decreases macroscopically; thus, reducing the effective contact area. Moreover, $P$ (surface $\rightarrow$ surface) in Eqs. (8) and (9) reduces microscopically as the activation volume is smaller with the larger interfacial gap as illustrated in Fig. 4(d). With an increase in the number of times of frictional contact, we can tell that the bonding numbers are increasing in a sub-linear way. This indicates that the wear and atomic transfer is less and approaches the saturation bonding numbers after several continuous frictional contacts.

For CoFs, all simulated values are within the reasonable engineering ranges as demonstrated in Fig. 7(b). However, all tribo-pairs possess a larger $\mathrm{CoF}$ with a separation of $18 \AA$. This illustrates the differences between the worst fretting friction conditions and the closest contact conditions. In our case, the closest contact condition exerts inter-atomic repulsion with such a small separation distance [54], which will slightly reduce the friction or heavy adhesion. On the other hand, if the separation is large enough, up to $24 \AA$, the contact area is suppressed when the interfacial distance increases beyond the possible effective shortrange atomic interaction range. Then, the $\mathrm{CoF}$ will be smaller for a larger surface separation.

The observation of the separation effects is important for MD simulations. As discussed, it will influence the fretting friction prediction accuracy and the optimization in applications. The worst fretting friction situation or other different situations needs to cater to the needs of real systems whether to choose the closest contact situation.

\section{Conclusions}

As the MD simulation indicates, $\mathrm{Al}$ shows a relatively high softness. This results in the increase in contact area, the atom/ion hetero-surface transfer, and the attachment. The easier atom/ion transfer and redistribution delays the friction force relaxation in fretting contact intervals. Because diamond and Si have no isotropic force response in the fretting process owing to their covalent bonds, they behave differently during the fretting process in the following ways. First, the contact area difference, the easiness of the atom/ion transfer, and the attachment contribute to different force responses. Second, owing to these factors, it is physically understandable that metal-metal contact, e.g., $\mathrm{Al}-\mathrm{Al}$, will have a larger $\mathrm{CoF}$.

The comparison between the CoF range and our simulated $\mathrm{CoF}$ proves the validity of our simulation for the continuous friction process at the fretting interface. In this sense, we should consider the importance of interfacial characteristics, e.g., microconfiguration and hardness, when analyzing the fretting process. The interfacial characteristics will lead to different force responses for $\mathrm{Al}-\mathrm{Al}$, diamonddiamond, and diamond-Si interfaces during friction contact intervals. As a result, the force investigation at the contact intervals must be considered in order to fully understand the whole continuous fretting 
process. Moreover, in simulations, the interface with a suitable separation is also a major factor for the actual replica of real systems. It is because the separation will affect the friction behavior of the observed fretting process.

In summary, a simulation study to reveal the microand macro-explanation for the different tribo-pairs has been conducted. Together with new theories that include energy barrier analyses, wear rate theories, and nanoscale friction laws, it is validated that our observations for these phenomena in the fretting processes can be generalized. Owing to the possible prominent force responses during contact intervals and the influence from the separation, the continuous frictional behavior on the fretting interfaces largely depends on the frictional intervals and the interface separation.

\section{Acknowledgements}

This study is financially supported by the National Natural Science Foundation of China (Grant Nos. 51575340, 51875343) and State Key Laboratory of Mechanical Systems and Vibrations Project (Grant No. MSVZD201912). We are grateful to Shi CHEN (Ph.D. student, Shanghai Jiao Tong University) and Junyu CHEN (Ph.D. student, University of California-Los Angeles) for their useful comments and proofreading.

Open Access This article is licensed under a Creative Commons Attribution 4.0 International License, which permits use, sharing, adaptation, distribution and reproduction in any medium or format, as long as you give appropriate credit to the original author(s) and the source, provide a link to the Creative Commons licence, and indicate if changes were made.

The images or other third party material in this article are included in the article's Creative Commons licence, unless indicated otherwise in a credit line to the material. If material is not included in the article's Creative Commons licence and your intended use is not permitted by statutory regulation or exceeds the permitted use, you will need to obtain permission directly from the copyright holder.

To view a copy of this licence, visit http://creativecommons.org/licenses/by/4.0/.

\section{References}

[1] Gay D. Composite Materials: Design and Applications. 3rd edn. Boca Raton (USA): CRC Press, 2015.

[2] Miracle D B. Metal matrix composites-from science to technological significance. Compos Sci Technol 65(15-16): 2526-2540 (2005)

[3] Njuguna J. Lightweight Composite Structures in Transport: Design, Manufacturing, Analysis and Performance. Waltham (USA): Woodhead Publishing, 2016.

[4] Javadi A, Pan S H, Li X C. Manufacturing of Al and Mg nanocomposite microparticles. Manuf Lett 17: 23-26 (2018)

[5] Noda T. Application of cast gamma TiAl for automobiles. Intermetallics 6(7-8): 709-713 (1998)

[6] Pan S H, Zhang Z N. Fundamental theories and basic principles of triboelectric effect: A review. Friction 7(1): 2-16 (2018)

[7] Ye F, Li Y S, Sun X Y, Yang Q Q, Kim C Y, Odeshi A G. CVD diamond coating on WC-Co substrate with Al-based interlayer. Surf Coat Technol 308: 121-127 (2016)

[8] Chandran M, Hoffman A. Diamond film deposition on WC-Co and steel substrates with a $\mathrm{CrN}$ interlayer for tribological applications. J Phys D: Appl Phys 49(21): 213002 (2016)

[9] Roberts E W. Space tribology: Its role in spacecraft mechanisms. J Phys D: Appl Phys 45(50): 503001 (2012)

[10] Miyoshi K, Sanders J H, Hager Jr C H, Zabinski J S, Wal R L V, Andrews R, Street Jr K W, Lerch B A, Abel P B. Wear behavior of low-cost, lightweight $\mathrm{TiC} / \mathrm{Ti}-6 \mathrm{Al}-4 \mathrm{~V}$ composite under fretting: Effectiveness of solid-film lubricant counterparts. Tribol Int 41(1): 24-33 (2008)

[11] Thompson P A, Robbins M O. Origin of stick-slip motion in boundary lubrication. Sci Wash 250(4982): 792-794 (1990)

[12] Dmitriev A I, Nikonov A Y, Österle W. MD sliding simulations of amorphous tribofilms consisting of either $\mathrm{SiO}_{2}$ or carbon. Lubricants 4(3): 24 (2016)

[13] Li X W, Joe M W, Wang A Y, Lee K R. Stress reduction of diamond-like carbon by $\mathrm{Si}$ incorporation: A molecular dynamics study. Surf Coat Technol 228(S1): S190-S193 (2013)

[14] Lan H Q, Kato T, Liu C. Molecular dynamics simulations of atomic-scale tribology between amorphous DLC and Si-DLC films. Tribol Int 44(11): 1329-1332 (2011)

[15] Chen Q, Xu F, Liu P, Fan H. Research on fractal model of normal contact stiffness between two spheroidal joint surfaces considering friction factor. Tribol Int 97: 253-264 (2016)

[16] Hu Y Z, Ma T B, Wang H. Energy dissipation in atomic-scale friction. Friction 1(1): 24-40 (2013) 
[17] Wang Z J, Ma T B, Hu Y Z, Xu L, Wang H. Energy dissipation of atomic-scale friction based on one-dimensional Prandtl-Tomlinson model. Friction 3(2): 170-182 (2015)

[18] Morita Y, Jinno S, Murakami M, Hatakeyama N, Miyamoto A. A computational chemistry approach for friction reduction of automotive engines. Int $J$ Engine Res 15(4): 399-405 (2014)

[19] Sha Z D, Sorkin V, Branicio P S, Pei Q X, Zhang Y W, Srolovitz D J. Large-scale molecular dynamics simulations of wear in diamond-like carbon at the nanoscale. Appl Phys Lett 103(7): 073118 (2013)

[20] Dong Y L, Li Q Y, Martini A. Molecular dynamics simulation of atomic friction: A review and guide. J Vac Sci Technol A 31(3): 030801 (2013)

[21] Si L N, Guo D, Luo J B, Lu X C. Monoatomic layer removal mechanism in chemical mechanical polishing process: A molecular dynamics study. J Appl Phys 107(6): 064310 (2010)

[22] Si L N, Guo D, Luo J B, Lu X C, Xie G X. Abrasive rolling effects on material removal and surface finish in chemical mechanical polishing analyzed by molecular dynamics simulation. J Appl Phys 109(8): 084335 (2011)

[23] Hu C Z, Bai M L, Lv J Z, Kou Z H, Li X J. Molecular dynamics simulation on the tribology properties of two hard nanoparticles (diamond and silicon dioxide) confined by two iron blocks. Tribol Int 90: 297-305 (2015)

[24] Gueye B, Zhang Y, Wang Y J, Chen Y F. Origin of frictional ageing by molecular dynamics simulation of a silicon tip sliding over a diamond substrate. Tribol Int 86: 10-16 (2015)

[25] Gosvami N N, Filleter T, Egberts P, Bennewitz R. Microscopic friction studies on metal surfaces. Tribol Lett 39(1): 19-24 (2010)

[26] Jansen L, Hölscher H, Fuchs H, Schirmeisen A. Temperature dependence of atomic-scale stick-slip friction. Phys Rev Lett 104(25): 256101 (2010)

[27] Pan S H, Yin N, Zhang Z N. Molecular dynamics simulation for continuous dry friction on fretting interfaces. $J$ Mech Eng 54(3): 82-87

[28] Plimpton S. Fast parallel algorithms for short-range molecular dynamics. J Comput Phys 117(1): 1-19 (1995)

[29] Ewen J P, Echeverri Restrepo S, Morgan N, Dini D. Nonequilibrium molecular dynamics simulations of stearic acid adsorbed on iron surfaces with nanoscale roughness. Tribol Int 107: 264-273 (2017)

[30] Boyd I D, Ketsdever A. Interactions between spacecraft and thruster plumes. J Spacecr Rockets 38: 380 (2001)

[31] Krishnan S. Processing of cotton-phenolic bearing retainers for optimum performance of spacecraft high-speed rotating systems. Tribol Trans 58(4): 704-711 (2015)

[32] Bhushan B. Introduction to Tribology. 2nd edn. New York (USA): John Wiley \& Sons, 2013.

[33] Kraska T. Molecular-dynamics simulation of argon nucleation from supersaturated vapor in the NVE ensemble. $\mathrm{J}$ Chem Phys 124(5): 054507 (2006)

[34] Jackson R L, Duvvuru R S, Meghani H, Mahajan M. An analysis of elasto-plastic sliding spherical asperity interaction. Wear 262(1-2): 210-219 (2007)

[35] Green I. An elastic-plastic finite element analysis of two interfering hemispheres sliding in frictionless contact. Phys Sci Int J 19(1): 1-34 (2018)

[36] Vijaywargiya R, Green I. A finite element study of the deformations, forces, stress formations, and energy losses in sliding cylindrical contacts. Int J Non-Linear Mech 42(7): 914-927 (2007)

[37] Liu Y L, Hua Y W, Jiang M, Xu M, Yu F, Chen J. Different orientations of molecular water on neutral and charged aluminium clusters $\mathrm{Al}_{17}{ }^{n \pm}(n=0-3)$. Eur Phys J D 67: 194 (2013)

[38] Daw M S, Foiles S M, Baskes M I. The embedded-atom method: A review of theory and applications. Mater Sci Rep 9(7-8): 251-310 (1993)

[39] Mendelev M I, Srolovitz D J, Ackland G J, Han S. Effect of Fe segregation on the migration of a non-symmetric $\Sigma 5$ tilt grain boundary in Al. J Mater Res 20(1): 208-218 (2005)

[40] Redkov A V, Osipov A V, Kukushkin S A. Molecular dynamics simulation of the indentation of nanoscale films on a substrate. Tech Phys Lett 42(6): 639-643 (2016)

[41] Abgaryan K, Mutigullin I. Theoretical investigation of the stability of defect complexes in silicon. Phys Status Solidi C 13(4): 156-158 (2016)

[42] Zhou N G, Wu X Y, Wei X Q, Zhou L, Wan Y P, Hu D. A molecular dynamics study of nucleation of dislocation in growth of silicon from melt. J Cryst Growth 443: 15-19 (2016)

[43] Cheng Y, Zhu P Z, Li R. The influence of vertical vibration on nanoscale friction: A molecular dynamics simulation study. Crystals 8(3): 129 (2018)

[44] Pan S H, Yin N, Zhang Z N. Time- \& load-dependence of triboelectric effect. Sci Rep 8(1): 2470 (2018)

[45] Kisiel M, Gnecco E, Gysin U, Marot L, Rast S, Meyer E. Suppression of electronic friction on $\mathrm{Nb}$ films in the superconducting state. Nat Mater 10(2): 119-122 (2011)

[46] Pan S H, Zhang Z N. Triboelectric effect: A new perspective on electron transfer process. J Appl Phys 122(14): 144302 (2017) 
[47] Wang A E, Gil P S, Holonga M, Yavuz Z, Baytekin H T, Sankaran R M, Lacks D J. Dependence of triboelectric charging behavior on material microstructure. Phys Rev Mater 1(3): 035605 (2017)

[48] Chung Y G, Lacks D J. Atomic mobility in strained glassy polymers: The role of fold catastrophes on the potential energy surface. J Polym Sci Part B Polym Phys 50(24): 1733-1739 (2012)

[49] Yu J X, Qian L M, Yu B J, Zhou Z R. Nanofretting behaviors of monocrystalline silicon (100) against diamond tips in atmosphere and vacuum. Wear 267(1-4): 322-329 (2009)

[50] Yu J X, Kim S H, Yu B J, Qian L M, Zhou Z R. Role of

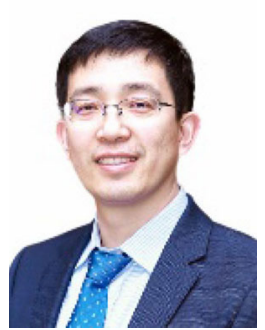

Zhinan ZHANG. He received his Ph.D. degree in 2011 from Shanghai Jiao Tong University, Shanghai, China. After that he was a post doctor in Shanghai Jiao Tong University. He is now working as an

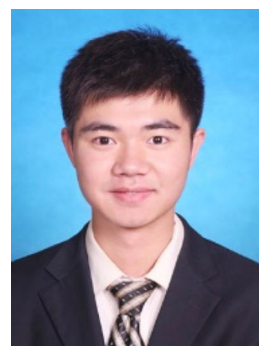

Shuaihang PAN. He received his B.S. in mechanical engineering and B.A. in German in 2016 from Shanghai Jiao Tong University, Shanghai, China. He is now working

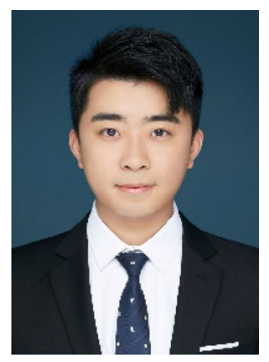

Nian YIN. He received his B.S. in mechanical engineering in 2017 from Shanghai Jiao Tong University, Shanghai, China. He is now a master student in School of Mechanical
Tribochemistry in Nanowear of single-crystalline silicon. ACS Appl Mater Interfaces 4(3): 1585-1593 (2012)

[51] Mo Y F, Turner K T, Szlufarska I. Friction laws at the nanoscale. Nature 457(7233): 1116-1119 (2009)

[52] Zheng X, Zhu H T, Tieu A K, Kosasih B. Roughness and lubricant effect on 3D atomic asperity contact. Tribol Lett 53(1): 215-223 (2014)

[53] Haynes W M. CRC Handbook of Chemistry and Physics, 95th Edition, 2014-2015: A Ready-Reference Book of Chemical and Physical Data. New York (USA): CRC Press, 2014.

[54] Feiler A A, Bergström L, Rutland M W. Superlubricity using repulsive van der Waals forces. Langmuir 24(6): 2274-2276 (2008)

associate professor in the School of Mechanical Engineering, Shanghai Jiao Tong University. His research interests include computational design and analysis of tribosystems, and theory and methods of design engineering and innovation.

as a graduate researcher at University of CaliforniaLos Angeles (UCLA) in pursuit of a Ph.D. degree. His research focuses on triboelectric effect, novel nanocomposites, and electrical/thermal transport phenomena.

Engineering, Shanghai Jiao Tong University. His research focuses on computational design of materials, triboelectric effect, and development of tribology testing instruments. 


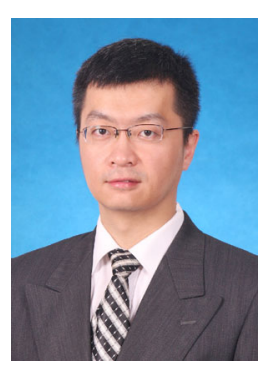

Bin SHEN. He received his Ph.D. degree in 2010 from Shanghai Jiao Tong University, Shanghai, China. After that he was a post doctor in Shanghai Jiao Tong University. He is now working as an associate

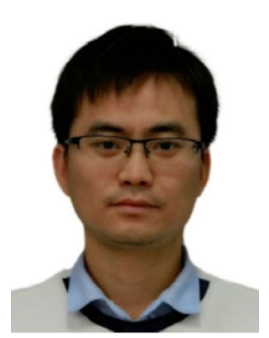

Jie SONG. He received his B.A. degree in physics in 2009 from Lanzhou University, China, and his Ph.D. degree in biophysics in 2014 from Aarhus University, Denmark. Then he worked as a post doctor in Aarhus University, Denmark and Emory University, US, for two years. professor in the School of Mechanical Engineering, Shanghai Jiao Tong University. His research interests include diamond and graphene based tribological and anti-corrosion coatings, and super-hard and smart coating for cutting tools.

He is now a distinguished research fellow from the Department of Instrument Science and Engineering in the School of Electronic Information and Electrical Engineering at Shanghai Jiao Tong University, China. His current research focuses on medical instrument development, high resolution dynamic imaging, single cell manipulation, and DNA nanotech for biomedical application. 